\title{
Biomarkers for hazard identification in humans
}

\author{
Helmut Bartsch ${ }^{1 *}$, Khelifa Arab ${ }^{1,2}$, Jagadeesan Nair ${ }^{1}$ \\ From First Lorenzo Tomatis Conference on Environment and Cancer \\ Turin, Italy. 4-5 June 2009
}

\begin{abstract}
Background: Oxidative stress enhances lipid peroxidation (LPO), which both are implicated in the promotion and progression stages of carcinogenesis, in particular under conditions of chronic inflammation and infections. Exocyclic etheno-DNA adducts, which are formed by LPO-products such as 4-hydroxy -2-nonenal, are strongly promutagenic DNA lesions.

Methods: The development of ultra-sensitive detection methods for etheno-adducts in human tissues, white blood cells $(\mathrm{WBC})$ and urine has provided evidence that these adducts are elevated in affected organs of cancerprone patients, probably acting as a driving force to malignancy.

Results: Two recent studies that yielded some new insights into disease causation are briefly reviewed:DNAdamage in WBC of mother-newborn child pairs, and lipid peroxidation derived DNA damage in patients with cancer-prone liver diseases. Our results indicate that biomonitoring of etheno-DNA adducts in humans are promising tools (i) to better understand disease aetiopathogenesis, allowing hazard identification(ii) to monitor disease progression and (iii) to verify the efficacy of chemopreventive and therapeutic interventions. Such clinical trials are warranted.
\end{abstract}

\section{Background}

Oxidative stress enhances lipid peroxidation (LPO), which both are implicated in the promotion and progression of chronic degenerative diseases (CDD), including carcinogenesis, in particular under conditions of chronic inflammation [1]. Common pathways of CDD involve biologically reactive oxygen and nitrogen species which can be generated by biochemical redox reactions and stress response enzymes such as lipoxygenases, cyclooxygenenase -2 and inducible nitric oxide synthase [2]. The resulting oxidative stress is currently implicated in over 100 human and animal CDD.Exocyclic ethenoDNA adducts, which are formed by LPO- products such as 4-hydroxy-2-nonenal (HNE), are strongly miscoding, causing specific point mutations. The development of ultra-sensitive detection methods for etheno-adducts in human tissues, WBC and urine provided evidence that these adducts are elevated in affected organs of cancer-

\footnotetext{
* Correspondence: helmut.bartsch@gmx.de

'Division of Toxicology and Cancer Risk Factors, German Cancer Research Center (DKFZ)Im Neuenheimer Feld 280, 69120 Heidelberg, Germany Full list of author information is available at the end of the article
}

prone patients, suggesting that such DNA damage can drive cells to malignancy [3].

A summary of studies that we have conducted is depicted in Fig. 1; detailed adduct levels and results ( published until 2007 ) are reviewed in reference [1]. Sources of DNA damage that significantly increased the physiological background of exocyclic etheno-DNA adducts were found to be known human cancer risk factors such as i) viral infections and chronic inflammatory processes, ii) genetic cancer predisposing diseases, iii) nutritional factors and iv) certain occupational exposures. The recent study in a Danish cohort of mother- newborn child pairs is separately dealt with.

In the following, recent studies in humans that yielded some new insights into disease causation are briefly reviewed: 1) DNA -damage in WBC DNA of mothernewborn child pairs [4], and 2) Lipid peroxidationderived DNA damage in patients with cancer-prone liver diseases [5]. Our results indicate that biomonitoring of etheno-DNA adducts in humans are promising tools (i) to better understand disease aetiopathogenesis, allowing hazard identification, especially when combined 


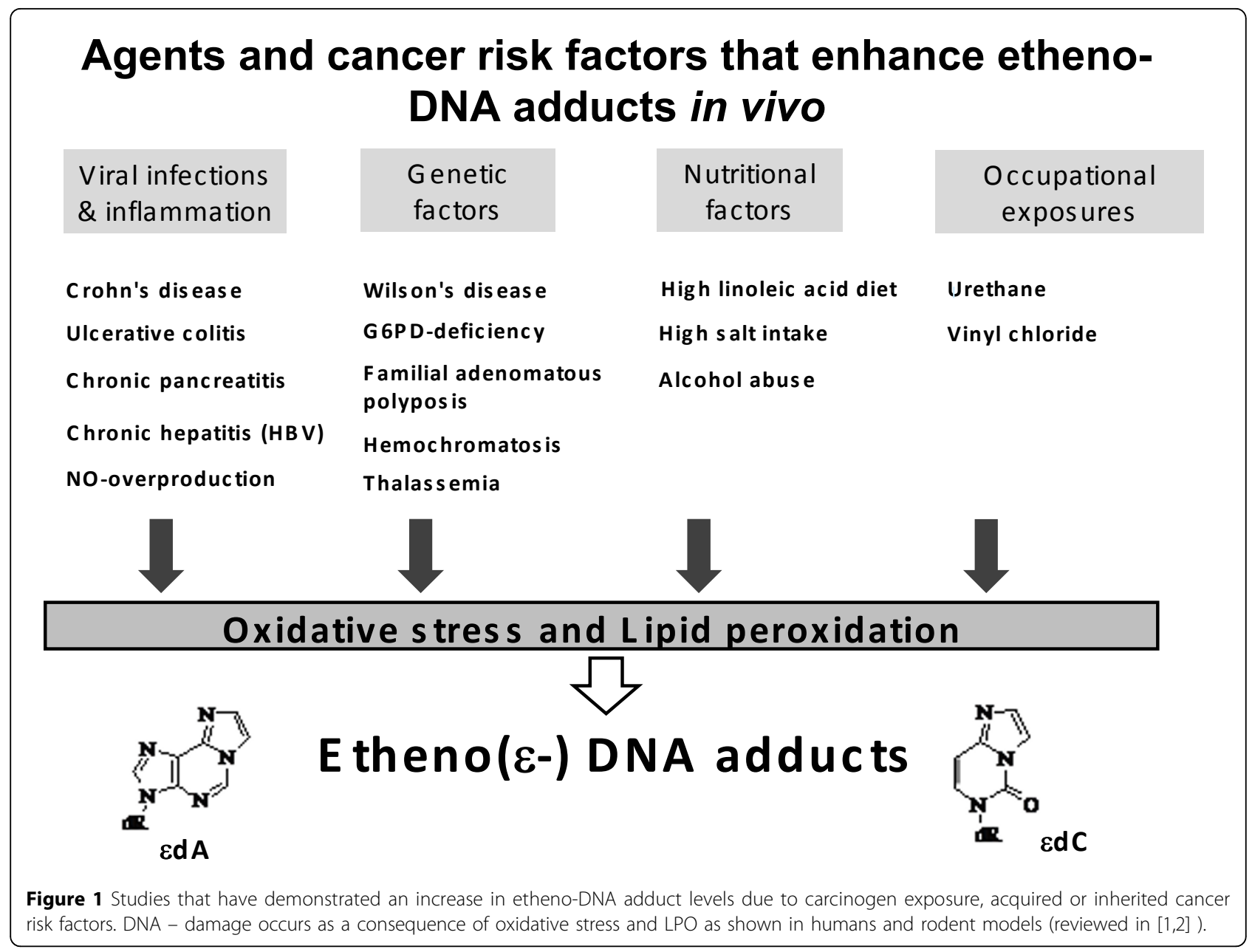

with genetic predisposition studies( e.g.as done in patients with metal storage diseases), (ii) to monitor diagnostic disease progression and (iii) to verify the efficacy of chemopreventive and therapeutic interventions. Such clinical trials are warranted and feasible due to the available non-invasive biomarkers we have developed.

\section{Methods}

We have developed ultrasensitive, specific and artifactfree detection methods using immunoenrichment that allow the biomonitoring of etheno DNA adducts in humans in vivo[1]. Protocols and technical details are described in [6]. The high sensitivity and specificity of the three methods (below) are due to the use of monoclonal antibodies. They recognize etheno-DNA adducts specifically, allowing a) their immunopurification and quantitation in human tissues, cells and in urine where the adducts are excreted ; and b) their semiquantative immunohistochemical detection in tissue mircoarrays.
-Adducts can now be quantified in tissue- and WBCDNA by an ultrasensitive immunoaffinity- ${ }^{32} \mathrm{P}$-postlabeling method.

-Etheno-adducts excreted in urine can be measured by an immuno-enrichment-HPLC-fluorescence method.

-Immunohistochemical staining in tissue sections allows to assess the prevalence of etheno-adducts in the nuclei of various cells and tissue types.

\section{Results}

Typical signature of exocyclic DNA damage measured in a cohort of mother-newborn child pairs

DNA damage is commonly thought to be involved in CDD causation, and its impact is particularly detrimental during fetal development. Hereby it is assumed that oxidative stress-related damage targets stem cells and their progenitors during differentiation, and that these early induced DNA lesions could cause hematopoietic disorders later in life. Within a multicenter study, we 
have analyzed 77 samples from mother-newborn child pairs to see if imprinting of DNA damage in mother child shows a similar pattern; it is known that cord blood is highly enriched with stem cells. Two etheno DNA-adducts, $\varepsilon \mathrm{dA}$ and $\varepsilon \mathrm{dC}$, were measured and their levels (expressed per $10^{9}$ parent nucleotides) in leucocytes varied from 400-9000, demonstrating that even the lowest levels could be reliably quantified by our detection method. In child cord blood adduct levels were found to be about two times lower than in maternal blood ,the difference being highly significant. Analysis of $\varepsilon \mathrm{dA}$ and $\varepsilon \mathrm{dC}$ levels in cord versus maternal blood WBC showed strong positive correlations $\left(\mathrm{R}^{2}=0.9, \mathrm{P}\right.$ $<0.00001)$. Our results confirm that the DNA damage observed in leucocytes, arises from LPO-derived reactive aldehydes such as HNE, and suggests that a typical signature of DNA damage is induced similarly in fetus and mother [4]. Prospective cohort studies have to reveal whether these exocyclic WBC-DNA adducts could serve as predictive hazard indicator for developing hematopoietic cancers and other disorders later in life. Attention should be focused on outliers with extremely high adduct levels that were also found in this study. Analyses by the study consortium whether specific exposures measured in these populations are related to etheno-adduct formation are in progress.

\section{Lipid peroxidation-derived DNA adducts: potential early hazard markers in patients with liver cancer -prone diseases}

Chronic alcohol abuse and hepatitis $B$, and $C$ virus (HBV, HCV) infection are causally associated with increased risk of developing hepatocellular carcinoma (HCC) which progresses through chronic hepatitis, fibrosis and cirrhosis. The disease progression is associated with intense oxidative stress in the liver, resulting in an overproduction of reactive oxygen /nitrogen species and LPO-products [7].

Our aims of the study were to ascertain whether $(i)$ etheno-adducts are formed in the liver of HCC-prone patients, (ii) etheno-adducts $(\varepsilon \mathrm{dA})$ areexcreted in urine and (iii) these adducts could serve as DNA damage (and putative risk) marker during viral -and alcohol -related liver cancer progression.

\section{Studies in ALD patients}

We analyzed $\varepsilon d A$ levels in DNA of needle liver biopsies obtained from European ALD patients diagnosed with alcohol-related hepatitis, fibrosis and cirrhosis using immunohistochemistry [8]. Cell nuclei positively stained for $\varepsilon \mathrm{dA}$ were counted and the \% prevalence was monitored. When compared to asymptomatic livers, the mean prevalence values were 16 times higher in biopsies from fibrosis/cirrhosis patients. This increase was comparable to that found in liver of patients with primary hemochromatosis, a genetically inherited disease highly predisposing to HCC. In European ALD patients, we also found in urine a 10 fold increased $\varepsilon \mathrm{dA}$-level as compared to healthy controls [9]. Thus high urinary $\varepsilon \mathrm{dA}$-excretion mirrors the massive DNA damage generated in the liver of ALD patients.

Excessive etheno-DNA adducts found in ALD patients are thought to be formed by the reaction of the major LPO product 4-hydroxy-2-nonenal (HNE) with nucleobases [5]. Confirmation for such a pathway in human liver in vivo was for the first time obtained by showing that protein- bound HNE and etheno adducts in ALD liver biopsies were strongly intercorrelated. In addition both markers correlated with CYP2E1 expression $\left(\mathrm{R}^{2}=0.9, \mathrm{P}=0.01\right)$

Our data support that ethanol-mediated induction of hepatic CYP2E1, leading to miscoding LPO-derived DNA lesions plays a central role in hepatocarcinogenesis, particularly in Caucasian ALD patients.

\section{Pilot studies in virus-infected patients}

As found in ALD patients, also subjects with inflammatory cancer - prone liver diseases caused by viral infection excreted high levels of $\varepsilon \mathrm{dA}$ in urine [9]. Thai patients with chronic hepatitis, liver cirrhosis and HCC due to HBV-infection had 20, 73 and 39 times higher urinary $\varepsilon \mathrm{dA}$-levels, respectively when compared with asymptomatic HBsAg-carriers. In over two thirds of European patients with $\mathrm{HBV}$ and $\mathrm{HCV}$-related liver disease, urinary $\varepsilon \mathrm{dA}$ levels were increased 6 to 10 fold [9].

From these studies we conclude that (i) high urinary \&dA-levels reflecting massive LPO-derived DNA-damage in vivo may contribute to the development HCC; (ii) \&dAmeasurments in urine and needle biopsies from target tissues in affected patients should be explored as a putative risk markers to follow malignant progression of inflammatory liver diseases and (iii) etheno adducts in urine may serve as non-invasive markers to assess to efficacy of chemopreventive and therapeutic interventions.

\section{Discussion}

Since more than two decades, starting under the directorship of Lorenzo Tomatis (from1982-1993) at IARC in Lyon we pursued the following hypothesis: Major risk factors for various epithelial human cancers are endogenously produced DNA reactive species, that arise from dietary imbalance, chronic inflammation, persistent infections and aberrant metal storage. These damaging agents escaped detection by conventional biomonitoring and epidemiological techniques. Therefore new approaches in molecular epidemiology are required that include non- invasive methods for identification of such DNA damage in humans. We contributed to this paradigm by studying modified DNA base adducts that are generated by reactions of DNA with LPO-products, such as HNE that yield 
etheno-adducts for which we have developed ultrasensitive detection methods.

We then investigated pathological conditions and known cancer risk factors that could significantly increase the physiological background of etheno-adduct levels. A summary of our previous studies is depicted in Fig. 1 . Our recent mother-newborn child study is not ncluded as we are unable to associate specific exposures/ life style factors with etheno adduct formation in these WBC. In this biomonitoring study we detected highly variable, and possibly cancerrelevant DNA damage in WBC-DNA of mother-newborn child pairs, conceivably derived from dietary imbalance and life style factors. One source of etheno adduct may be explained by our previous findings that high dietary omega6 polyunsaturated fatty acid (linoleic acid) intake drastically increased the formation of etheno-DNA adducts in white blood cells of female but not of male subjects [10]. In our mother -child study the DNA damage observed in maternal WBC-DNA was found to be very similar in DNA of newborns. In addition (epi)genome - environment interactions need to be further studied, so to allow better disease risk evaluations and predictions in children.

Our other investigations in cancer prone patients found progressively increasing DNA damage in preneoplastic liver tissue, due to alcohol abuse and viral infections, both causing chronic inflammation cascades that impose persistent oxidative stress in this organ.As published recently, tobacco smoking and UV- light induced many thousands of sequence alterations and specific signatures of somatic mutations in human lung cancer and melanoma [11,12]. Exocyclic LPO- derived DNA-lesions are likely to contribute to the mutation load reported to occur in human tumors.

Outlook: Since the discontinuation of our research project at DKFZ, there are a number of issues in relation to the utility of etheno adducts as biomarkers for hazard identification that need to be resolved:

Only three studiesin humans( Fig.1) have shown a positive relationship between etheno adduct formation and exposure to specific agents: Linoleic acid(omega-6 PUFA), sodium chloride and alcohol.

In contrast to rodent studies, only a few dose-reponse curves but mostly high $v s$ low dose (intake) comparisons were done. Etheno adducts in urine of Japanese subjects living in a high risk area for gastric cancer were positively associated with the excretion sodium chloride ( a risk factor for gastric cancer) and omega-6 polyunsaturated fatty acid intake .

The time window was mostly investigated in rodent cancer models. In cancer prone-patients etheno adduct levels progressively increased in target organs, being highest in preneoplastic lesions with subsequent decline in the tumor tissue, the same time course as also seen in rodents.
The diagnostic and prognostic value of etheno adducts ,measured in needle tissue biopsies, white blood cells and as urinary excretion products needs to be established. Initial attempts to develop an inexpensive and simple kit for adduct analysis to allow easy application in clinical and epidemiologial studies, was not successful. Ultrasensitive, specific mass- spectrometric methods that have become available, should augur progress in this validation process, allowing high throughput work flow for large cohort analyses.

\section{Conclusions}

From consistent results obtained in our past conducted studies ( Fig.1) we conclude:

(i) Etheno- adducts,stable DNA damage markers are elevated by various cancer risk factors that impose oxidative/ nitrosative stress and LPO on a cell.

(ii) Etheno-adducts progressively increased in many target organs and preneoplastic lesions of cancer pronepatients and rodents.

(iii) LPO-derived DNA adducts appear to be one of the causes of mutations and genomic instability that drives carcinogenesis, offering promising markers for quantifying increasing loads of promutagenic DNA damage in early stages of carcinogensis.

(iv) Etheno adducts in should be useful for etiology studies on cancers with ill-defined mechanisms, such as childhood malignancies: High DNA damage was found in some newborn child -mother pairs for which the cause is unknown.

From our findings in liver cancer prone patients we conclude:

(i) High urinary $\varepsilon d A$-levels reflecting massive LPOderived DNA damage in the affected liver, may contribute to the initiation and progression of HCC.

(ii) Measurements of etheno adducts in urine and in needle liver biopsies should be explored as a putative risk markers to follow the malignant disease progression in high risk patients patients and due to ALD and the viral infections.

(iii) Etheno adducts as biomarkers hold promise to assess the efficacy of chemopreventive and therapeutic interventions and such clinical/biomonitoring trials are warranted.

\section{List of abbreviations}

हdA: 1,N6 -ethenodeoxyadenosine; $\varepsilon d C: 3, N^{4}$-ethenodeoxycytine; ALD: alcohol-related disease; HBV: hepatitis B virus; HCV: hepatitis C virus; HCC: hepatocellar carcinoma; HNE: 4-hydroxy-2-nonenal; CYP2E1: Cytochrome 2E1; ROS: reactive oxygen species; LPO: lipid peroxidation; CDD: chronic degenerative diseases.

\section{Acknowledgments}

DNA adduct studies referred to Figure. 1 were conducted by great number of distinguished researchers at the German Cancer Research Center (DKFZ) 
in Heidelberg, Germany and at IARC in Lyon, France as well by many external collaborators; all their contributions are greatly acknowledged. Research was supported by the Division of Toxicology and Cancer risk factors, DKFZ and the European Union Integrated Project Newgeneris, $6^{\text {th }}$ Framework Program (Food-DC-2005-016320); KA was a recipient of the European Union-supported Newgeneris Network fellowship.

This paper is dedicated to late Dr. Jagadeesan Nair (1953-2007), a recognized pionier in the field of cancer biomarkers (cf Carcinogenesis 2008; 29: 887-8)

\section{Competing interest}

The authors declare that they have no competing financial or non-financial interests

\section{Author's contribution}

Helmut Bartsch was Division Head at DKFZ (until 2008) and conducted molecular epidemiology studies in which biomarkers for endogenous DNA damage were integrated.

Jagadeesan Nair developed ultra-sensitive and specific methods for etheno DNA adduct analysis which were subsequently applied in mechanistic and clinical studies.

Khelifa Arab was a visiting scientist at DKFZ and as co-principal investigator he analyzed WBC-DNA damage in mother-newborn child pairs within the EU-Newgeneris Project.

This article has been published as part of Environmental Health Volume 10 Supplement 1, 2011: Proceedings of the First Lorenzo Tomatis Conference on Environment and Cancer. The full contents of the supplement are available online at http://www.ehjournal.net/supplements/10/s1.

\section{Author details}

${ }^{1}$ Division of Toxicology and Cancer Risk Factors, German Cancer Research Center (DKFZ)Im Neuenheimer Feld 280, 69120 Heidelberg, Germany. ${ }^{2}$ Division of Epigenomics and Cancer Risk Factors, German Cancer Research Center (DKFZ)Im Neuenheimer Feld 280, 69120 Heidelberg, Germany.

Published: 5 April 2011

\section{References}

1. Nair U, Bartsch H, Nair J: Lipid peroxidation-induced DNA damage in cancer-prone inflammatory diseases: a review of published adduct types and levels in humans. Free Radic Biol Med 2007, 43:1109-1120.

2. Bartsch $H$, Nair J: Chronic inflammation and oxidative stress in the genesis and perpetuation of cancer: role of lipid peroxidation, DNA damage, and repair. Langenbecks Arch Surg 2006, 391:499-510.

3. Nair J, Barbin A, Guichard Y, Bartsch H: 1,N6-ethenodeoxyadenosine and 3, N4-ethenodeoxycytine in liver DNA from humans and untreated rodents detected by immunoaffinity/32P-postlabeling. Carcinogenesis 1995 , 16:613-617.

4. Arab K, Pedersen M, Nair J, Meerang M, Knudsen LE, Bartsch H: Typical signature of DNA damage in white blood cells: a pilot study on etheno adducts in Danish mother-newborn child pairs. Carcinogenesis 2009, 30:282-285.

5. Wang Y, Millonig G, Nair J, Patsenker E, Stickel F, Mueller S, Bartsch H, Seitz HK: Ethanol-induced cytochrome P4502E1 causes carcinogenic etheno-DNA lesions in alcoholic liver disease. Hepatology 2009, 50:453-461.

6. Nair J, Nair U, Sun X, Wang Y, Arab K, Bartsch H: Ultrasensitive 32PPostlabelling and immunohistochemical detection methods for quantifying etheno-DNA adducts in human tissues; white blood cells and urine. In Human Press Inc. Totowa NJ;V. Didenko 2010:

7. Seitz HK, Stickel F: Molecular mechanisms of alcohol-mediated carcinogenesis. Nat Rev Cancer 2007, 7:599-612.

8. Frank A, Seitz HK, Bartsch H, Frank N, Nair J: Immunohistochemical detection of 1,N6-ethenodeoxyadenosine in nuclei of human liver affected by diseases predisposing to hepato-carcinogenesis. Carcinogenesis 2004, 25:1027-1031.

9. Nair J, Srivatanakul P, Haas C, Jedpiyawongse A, Khuhaprema T, Seitz HK, Bartsch $\mathrm{H}$ : High urinary excretion of lipid peroxidation-derived DNA damage in patients with cancer-prone liver diseases. Mutat Res 2010, 683:23-28.

10. Nair J, Vaca CE, Velic I, Mutanen M, Valsta LM, Bartsch $H$, et al: High dietary omega- 6 polyunsaturated fatty acids drastically increase the formation of etheno-DNA base adducts in white blood cells of female subjects. Cancer Epidemiol Biomarkers Prev 1997, 8:597-601.

11. Pleasance ED, Stephens PJ, O'Meara S, McBride DJ, Meynert A, Jones D, Lin ML, Beare D, Lau KW, Greenman C, et al: A small-cell lung cancer genome with complex signatures of tobacco exposure. Nature 2010, 463:184-190.

12. Pleasance ED, Cheetham RK, Stephens PJ, McBride DJ, Humphray SJ, Greenman CD, Varela I, Lin ML, Ordonez GR, Bignell GR, et al: A comprehensive catalogue of somatic mutations from a human cancer genome. Nature 2010, 463:191-196.

13. /12 /J Nair, CE Vaca, I Velic, M Mutanen, LM Valsta, H Bartsch: High dietary omega- 6 polyunsaturated fatty acids drastically increase the formation of etheno-DNA adducts in white blood cells of female subjects. Cancer Epidemiol. Biomarkers Prev 1997, 6:597-601.

doi:10.1186/1476-069X-10-S1-S11

Cite this article as: Bartsch et al:: Biomarkers for hazard identification in humans. Environmental Health 2011 10(Suppl 1):S11.

\section{Submit your next manuscript to BioMed Central and take full advantage of:}

- Convenient online submission

- Thorough peer review

- No space constraints or color figure charges

- Immediate publication on acceptance

- Inclusion in PubMed, CAS, Scopus and Google Scholar

- Research which is freely available for redistribution

Submit your manuscript at www.biomedcentral.com/submit 